\title{
O Sistema Integrado de Gestão de Atividades Acadêmicas (SIGAA) na pós-graduação da UFPA: estudo de caso no NAEA
}

\section{The integrated system for the management of academic activities (SIGAA) of graduate programs at UFPA: a case study on NAEA}

José Nilberlanio Vieira - Mestrado em Planejamento do Desenvolvimento, pelo Programa de Pós-Graduação em Desenvolvimento Sustentável do Trópico Úmido da Universidade Federal do Pará (PPGDSTU/UFPA). E-mail: nilber2004@gmail.com

Hisakhana Pahoona Corbin-Doutor em Ciências, área de concentração em Desenvolvimento Socioambiental, pela Universidade Federal do Pará (UFPA). Professor e Pesquisador em População e Desenvolvimento, do Núcleo de Altos Estudos Amazônicos (NAEA/ UFPA). E-mail: hisacorbin@hotmail.com

\section{Resumo}

Este artigo procura analisar o Sistema Integrado de Gestão de Atividades Acadêmicas (SIGAA) e sua relação com a gestão pública no âmbito organizacional de programas de pós-graduação da Universidade Federal do Pará (UFPA). Para tanto, realizou-se um estudo de caso com a utilização de métodos mistos (qualitativo e quantitativo), no Programa de Pós-Graduação em Desenvolvimento Sustentável do Trópico Úmido (PPGDSTU) oferecido pelo Núcleo de Altos Estudos Amazônicos (NAEA) da UFPA. Os pesquisadores investigaram a utilidade do SIGAA para o gerenciamento acadêmico e administrativo nesse programa na opinião de docentes, discentes e servidores técnicoadministrativos. Os resultados apontaram que o SIGAA tem se mostrado útil para o gerenciamento acadêmico-administrativo dos cursos, sendo capaz de fornecer dados e informações que contribuem para o processo de tomada de decisão. Porém, para alguns discentes, o SIGAA poderia ser mais bem explorado, sobretudo por parte de alguns docentes que resistem em explorar todas as potencialidades presentes no sistema.

\section{Palavra-chave}

Tecnologia da Informação e Comunicação (TIC). Gestão Universitária. Universidade Federal do Pará (UFPA). Sistema Integrado de Gestão de Atividades Acadêmicas (SIGAA). Núcleo de Altos Estudos Amazônicos (NAEA).

\begin{abstract}
This article seeks to analyze the Integrated Academic Activities Management System (SIGAA) and its relationship to public management in the organizational scope of graduate programs at the Federal University of Pará (UFPA). For this motive, a case study was conducted using mixed methods (qualitative and quantitative), in the Postgraduate Program in Sustainable Development of the Humid Tropics (PPGDSTU) offered by the Center for Advanced Amazonian Studies (NAEA) at UFPA. The researchers investigated the usefulness of SIGAA for academic and administrative management in this program based on the opinions of professors, students and technical-administrative staffers. The results showed that SIGAA was useful for academic-administrative management of courses by furnishing data and information that contributed to decision-making. However, according to some students, SIGAA could be better explored, particularly by some professors who are reluctant to explore all capabilities of the system.
\end{abstract}

\section{Keywords}

Information and Communication Technology (ICT). University Management. Federal University of Pará (UFPA). Integrated System for the Management of Academic Activities (SIGAA). Center for Advanced Amazonian Studies (NAEA). 


\section{INTRODUÇÃO}

No mundo atual, a tecnologia da informação e comunicação (TIC) desempenha um papel central em diversos aspetos em educação superior, mas ainda existe uma série de desafios que limitam o uso, a adaptação e a maximização das potencialidades entre as diversas categorias de usuários (DINTOE, 2018).

$\mathrm{Na}$ literatura consultada, revela-se importante conhecer as transformações tecnológicas pelas quais a gestão da educação superior no Brasil vem passando nos últimos anos, sobretudo devido à implementação de modernos sistemas de informação para o gerenciamento da informação acadêmica e administrativa em âmbito universitário (FARIAS FILHO; VILHENA; NASCIMENTO, 2014; VILHENA, 2011; YOSHINO; RAMOS, 2015).

Neste sentido, o foco deste artigo é analisar como o Sistema Integrado de Gestão de Atividades Acadêmicas (SIGAA), enquanto uma Tecnologia da Informação e Comunicação (TIC), tem contribuído para a modernização da gestão pública no âmbito administrativo e acadêmico do Programa de PósGraduação em Desenvolvimento Sustentável do Trópico Úmido (PPGDSTU), de caráter interdisciplinar, pertencente à Universidade Federal do Pará (UFPA).

As seções a seguir apresentam a metodologia da pesquisa, o sistema de informação em universidades brasileiras, os resultados e a discussão. Em seguida, apresentam-se as conclusões e as recomendações.

\section{METODOLOGIA}

Este estudo de caso foi realizado por meio de uma combinação dos métodos quantitativo e qualitativo (método misto), pois, conforme Creswell (2007), a técnica de métodos mistos emprega estratégias de investigação que "envolvem coleta tanto de informações numéricas como de texto, de forma que o banco de dados final representa tanto informações quantitativas como qualitativas" (CRESWELL, 2007, p. 35). Além disso, foram realizados levantamentos e análise bibliográfica, objetivando preencher lacunas e ampliar estudos anteriores. Para a coleta de dados, foram utilizados instrumentos como: questionários e entrevistas abertas e semiestruturadas, que foram aplicados com 11 docentes, 33 discentes e 01 servidora técnico-administrativa. Foram consultados também arquivos/ registros. Os dados foram analisados utilizando-se a técnica de estatística descritiva com apoio de Microsoft Excel. 
Optou-se por iniciar a pesquisa de campo com a técnica qualitativa, com a qual se procurou ter maior contato com os participantes, permitindo maior grau para mudanças nos procedimentos metodológicos e conceituais, neste caso considerando situações não previstas.

\section{OS SISTEMAS DE INFORMAÇÃO EM UNIVERSIDADES BRASILEIRAS E A IMPLEMENTAÇÃO DO SISTEMA INTEGRADO DE GESTÃO DE ATIVIDADES ACADÊMICAS (SIGAA) NA UNIVERSIDADE FEDERAL DO PARÁ (UFPA)}

A Universidade Federal do Pará é constituída por 12 campi distribuídos em toda a extensão do Estado (UFPA EM NÚMEROS, 2016), sendo o Núcleo de Altos Estudos Amazônicos (NAEA) uma unidade acadêmica pertencente à UFPA, responsável pelos cursos de pós-graduação stricto sensu de Mestrado e Doutorado e de Especialização lato sensu, de caráter interdisciplinar (NÚCLEO DE ALTOS ESTUDOS AMAZÔNICOS, 2017). No NAEA, estão localizados os cursos de mestrado em Planejamento do Desenvolvimento, mestrado em Gestão Pública e doutorado em Desenvolvimento Socioambiental. Na última avaliação quadrienal (2013-2016) da Coordenação de Aperfeiçoamento de Pessoal de Nível Superior (CAPES), o PPGDSTU, foco deste estudo, foi avaliado com o conceito 6, passando a figurar entre os cursos de excelência no país, notadamente na região Norte.

Sabe-se que a Gestão Pública universitária tem vivenciado várias mudanças nos últimos tempos, e esta expressão não abarcaria apenas a migração de pensamento, mas também do ensino e pesquisa universitária (PORCIUNCULA, 2001).

Especificamente, quanto ao aspecto dos avanços tecnológicos, percebe-se que o Estado, ainda que por força de lei, tem buscado formas de se inserir no contexto dessas mudanças. Assim, tem adotado processos e políticas públicas de interação e inserção do cidadão no cotidiano do governo, visando à transparência das ações realizadas em favor do cidadão e enfocando a modernização da gestão pública no âmbito de suas atividades (PORCIUNCULA, 2001; DIAS, 2008).

Diversos autores concordam que quando uma organização como uma Instituição de Ensino Superior (IES) toma a decisão pela utilização de sistemas de informação, ela espera obter diversos benefícios, como a integração, o incremento das possibilidades de controle sobre os processos da organização, a atualização tecnológica, a redução de custos de informática e o acesso a 
informações de qualidade em tempo real para a tomada de decisões sobre toda a organização (YOSHINO; RAMOS, 2015; PORTO; MAGALHÃES, 2014). Além disso, a adoção de um SI por uma organização geralmente produz mudanças importantes, alterando, em certos aspectos, a forma como as pessoas desempenham seu trabalho (PINTO et al., 2019).

Neste sentido, entende-se que a gestão das Instituições Federais de Ensino Superior (IFES) parece especialmente complexa, pois envolve atividades administrativas e de cunho acadêmico (ensino, pesquisa e extensão), as quais possuem processos de trabalho diferenciados. Por outro lado, o setor público vem cada vez mais sofrendo pressões internas e externas com vistas a melhorar seu desempenho, tendo em vista as próprias demandas da sociedade, que requerem um serviço mais ágil e condizente com os impostos que os cidadãos pagam (YOSHINO; RAMOS, 2015; VIEIRA; CORBIN, 2016).

Destaque-se que as novas tecnologias estão cada vez mais presentes no cotidiano da sociedade (CASTELLS, 1999), bem como das IES, e as ferramentas das Tecnologias da Informação e Comunicação podem favorecer novas formas e espaços de acesso à informação. Isto acontece porque "o sistema de informação acadêmico, ou simplesmente sistema acadêmico, é a base para uma boa gestão da universidade como um todo" (ROCHA NETO; LIMA, 2009, p. 1).

Dentre as experiências que podem ser citadas em outras universidades brasileiras quanto à adoção de softwares para gerenciamento acadêmico, há a da Universidade Federal de Santa Maria, que desenvolveu o software SIE. Este é um dos sistemas mais utilizados e foi adotado por algumas IES, inclusive a UFPA, a partir do ano de 2006.

Outro exemplo é a Universidade Federal do Ceará (UFC), que adotou, na década de 1990, o Sistema de Automação Universitária (SAU), composto por módulos administrativos e de gestão de pessoal e de atividades didáticas, além da gestão de bibliotecas.

Para além desses sistemas citados, diversas universidades brasileiras vêm adotando gradativamente o SIGAA. Neste sentido, em 2009, a Universidade Federal do Rio Grande do Norte, idealizadora do SIGAA, firmou termos de cooperação técnica na área de Tecnologia da Informação com algumas universidades brasileiras, dentre as quais a UFPA, cujo objetivo com a aquisição do SIGAA tem sido contribuir para melhorar o trabalho, promover interação e integração entre os diversos setores e processos de trabalho na instituição.

Houve, então, a decisão de que seria adquirido um sistema integrado já desenvolvido, a fim de integrar todas as unidades da organização, realizando 
cruzamento de informações e possibilitando a redução de retrabalho e inconsistências, bem como a geração de informações com maior rapidez (YOSHINO, 2010), e ainda a necessidade de modernização da gestão inserida nas diretrizes da UFPA.

Assim, a UFPA optou pelo SIGAA, um Sistema que informatiza os procedimentos da área acadêmica. Esse sistema possui os seguintes módulos: graduação, pós-graduação (stricto e lato sensu), ensino técnico, ensinos médio e infantil, submissão e controle de projetos e bolsistas de pesquisa, submissão e controle de ações de extensão, submissão e controle dos projetos de ensino (monitoria e inovações), registro e relatórios da produção acadêmica dos docentes, atividades de ensino a distância e um ambiente virtual de aprendizado denominado Turma Virtual (UNIVERSIDADE FEDERAL DO PARÁ, 2011). Uma característica importante desse sistema é a disponibilização de diversos tipos de relatórios - técnicos e gerenciais - em tempo real, que são de extrema importância nas tomadas de decisão dos gestores e usuários.

Além disso, qualquer usuário poderá acessar o SIGAA de qualquer lugar do mundo, bastando para isso estar conectado à internet (UNIVERSIDADE FEDERAL DO PARÁ, 2011). Isso facilita a vida dos usuários, notadamente os discentes, que não necessitam mais se deslocar até a Universidade para resolver determinadas questões referentes à sua vida acadêmica, tais como emissão de histórico, declarações, realização de matrícula on-line etc.

Destarte, a gestão dos sistemas de informações deve fundamentar um planejamento estratégico que apresente diretrizes que não estejam ligadas apenas aos aspectos legais, mas sim às práticas gerenciais que viabilizem decisão dos administradores (DÍAZ et al., 2014). Isso é particularmente importante quando se leva em consideração também os programas de pós-graduação stricto sensu, como o Programa de Pós-Graduação em Desenvolvimento Sustentável do Trópico Úmido, do Núcleo de Altos Estudos Amazônicos, da Universidade Federal do Pará (PPGDSTU/NAEA/UFPA), o qual é objeto de análise neste artigo.

\section{RESULTADOS E DISCUSSÃO: SIGAA NO PPGDSTU/NAEA}

Foram realizados um estudo de caso e um resumo das principais perguntas feitas na entrevista com docentes, discentes e servidores técnico-administrativos do PPGDSTU/NAEA (Quadro 1). 
Quadro 1 - Resumo de resultados agregados do PPGDSTU/NAEA

\begin{tabular}{|c|c|c|c|c|}
\hline Perguntas-chave & $\begin{array}{c}\text { № Docentes } \\
\text { PPGDSTU/ } \\
\text { NAEA } \\
(\mathrm{n}=11)\end{array}$ & $\begin{array}{c}\text { № Discentes } \\
\text { PPGDSTU/ } \\
\text { NAEA } \\
(\mathrm{n}=33)\end{array}$ & $\begin{array}{c}\text { No }{ }^{\circ} \text { T.A. } \\
\text { PPGDSTU/ } \\
\text { NAEA } \\
(\mathrm{n}=1)\end{array}$ & $\begin{array}{c}\text { Total } \\
(n=45)\end{array}$ \\
\hline \multicolumn{5}{|c|}{ Como você avalia o sistema SIGAA? } \\
\hline Ótimo & 9,1 & 18,2 & - & 15,5 \\
\hline Bom & 63,6 & 81,8 & 100,0 & 77,8 \\
\hline Ruim & 27,3 & - & - & 6,7 \\
\hline Total & 100,0 & 100,0 & 100,0 & 100,0 \\
\hline \multicolumn{5}{|c|}{ Você está satisfeito com o SIGAA? } \\
\hline Sim & 72,7 & 90,9 & 100,0 & 86,7 \\
\hline Não & 27,3 & 9,1 & - & 13,3 \\
\hline Total & 100,0 & 100,0 & 100,0 & 100,0 \\
\hline \multicolumn{5}{|c|}{ Você tem dificuldade em utilizar o SIGAA? } \\
\hline Sim & 54,5 & 3,0 & - & 15,6 \\
\hline Não & 45,5 & 97,0 & 100,0 & 84,4 \\
\hline Total & 100,0 & 100,0 & 100,0 & 100,0 \\
\hline \multicolumn{5}{|c|}{ Com que frequência você utiliza o SIGAA? } \\
\hline Diariamente & - & 3,0 & 100,0 & 4,4 \\
\hline Semanalmente & 18,2 & 42,3 & - & 35,5 \\
\hline Mensalmente & 45,4 & 24,3 & - & 28,9 \\
\hline Eventualmente & 36,4 & 30,4 & - & 31,2 \\
\hline Total & 100,0 & 100,0 & 100,0 & 100,0 \\
\hline \multicolumn{5}{|c|}{ Você considera que o SIGAA facilita sua vida e/ou trabalho? } \\
\hline Sim & 63,6 & 94,0 & 100,0 & 86,7 \\
\hline Não & 36,4 & 6,0 & - & 13,3 \\
\hline Não faz diferença & - & - & - & - \\
\hline Total & 100,0 & 100,0 & 100,0 & 100,0 \\
\hline
\end{tabular}

Fonte: Pesquisa de campo (2017).

\subsection{DO CORPO TÉCNICO-ADMINISTRATIVO}

A servidora técnico-administrativa do PPGDSTU/NAEA que respondeu ao questionário da presente pesquisa avalia o SIGAA como um sistema bom, estando satisfeita com este. Além disso, possui um bom domínio de informática em geral e não relata dificuldade em utilizar o SIGAA, pois, segundo ela, "muitas atividades do SIGAA são autoexplicativas”. Em suas palavras, o SIGAA “é um sistema eficaz e ajuda bastante nas atividades da secretaria acadêmica". Contudo, 
ressaltou que "é necessário aprimorar algumas opções, como os tipos de relatórios, declarações". A servidora recebeu treinamento para a utilização do SIGAA no âmbito acadêmico, entretanto, conforme suas palavras, "as informações repassadas no treinamento são insuficientes” (informações verbais) ${ }^{1}$.

Tal assertiva faz sentido quando se analisa a complexidade do trabalho desenvolvido em uma secretaria de pós-graduação stricto sensu, pois o volume de trabalho é bastante expressivo e as demandas diárias são bastante diversas. Estas questões não são exploradas em profundidade em um treinamento. Entendese que é na prática do dia a dia que ocorrem os desafios com a utilização do SIGAA e o adequado gerenciamento das informações. Isso é corroborado por Vieira (2018), ao afirmar que no dia a dia de uma secretaria de pós-graduação são vivenciadas diversas situações que requerem o domínio de técnicas para adequadamente gerenciar os processos de trabalho e apresentar resultados para a gestão acadêmica e administrativa de modo geral, visto que as IES são espaços complexos e diferenciados (BURIGO et al., 2015).

Neste aspecto, a adequada instrumentalização dos sistemas de informação disponíveis, a exemplo do SIGAA, pode contribuir para uma melhora nos processos de trabalho desenvolvidos em uma secretaria de pós-graduação stricto sensu de uma IES.

Sobre a frequência de utilização do SIGAA, a servidora respondeu que o utiliza diariamente, pois "O trabalho na secretaria acadêmica é diretamente ligado ao SIGAA” (informação verbal) ${ }^{2}$. Indagada se o SIGAA facilita sua vida e/ou trabalho, a servidora respondeu que sim, pois ele contribui para aumentar a eficiência e eficácia do trabalho no NAEA e reduzir erros humanos na elaboração de documentos. No que tange às recomendações para melhoria da utilização do SIGAA, a servidora listou as seguintes:

- Uma vez que as declarações referentes a bancas são emitidas pelo SIGAA, as ATAS também poderiam ser emitidas pelo sistema.

- Conexão das informações do sistema junto ao site de cada Pós. Assim como é encaminhada a informação via e-mail, poderia ser postada a mesma informação na página oficial da Pós (informação verbal) ${ }^{3}$.

As recomendações citadas são importantes e o Centro de Tecnologia da Informação e Comunicação (CTIC/UFPA) poderia realmente trabalhar no sentido de implantar tais melhorias no SIGAA, a fim de aprimorá-lo cada vez mais

\footnotetext{
Informação concedida pela servidora técnico-administrativa do PPGDSTU/NAEA, em 15 de maio de 2017.

2 Informação concedida pela servidora técnico-administrativa, em 15 de maio de 2017.

3 Informação concedida pela servidora técnico-administrativa do NAEA/UFPA, em 15 de maio de 2017.
} 
como uma ferramenta auxiliar no gerenciamento acadêmico e administrativo de cada PPG, em específico, e em todos os cursos da UFPA, de modo geral, uma vez que, segundo o diretor do CTIC/UFPA, a universidade tem acesso ao códigofonte $^{4}$ do sistema e pode adaptá-lo à realidade local.

\subsection{DO CORPO DOCENTE}

A pesquisa realizada com os docentes do PPGDSTU/NAEA revelou que apenas $9 \%$ dos entrevistados consideram o SIGAA ótimo, enquanto $27 \%$ o consideram ruim e $64 \%$ o consideram um bom sistema. Os resultados são apresentados no Gráfico 1.

Gráfico 1 - Avaliação do SIGAA - docentes entrevistados no PPGDSTU/NAEA

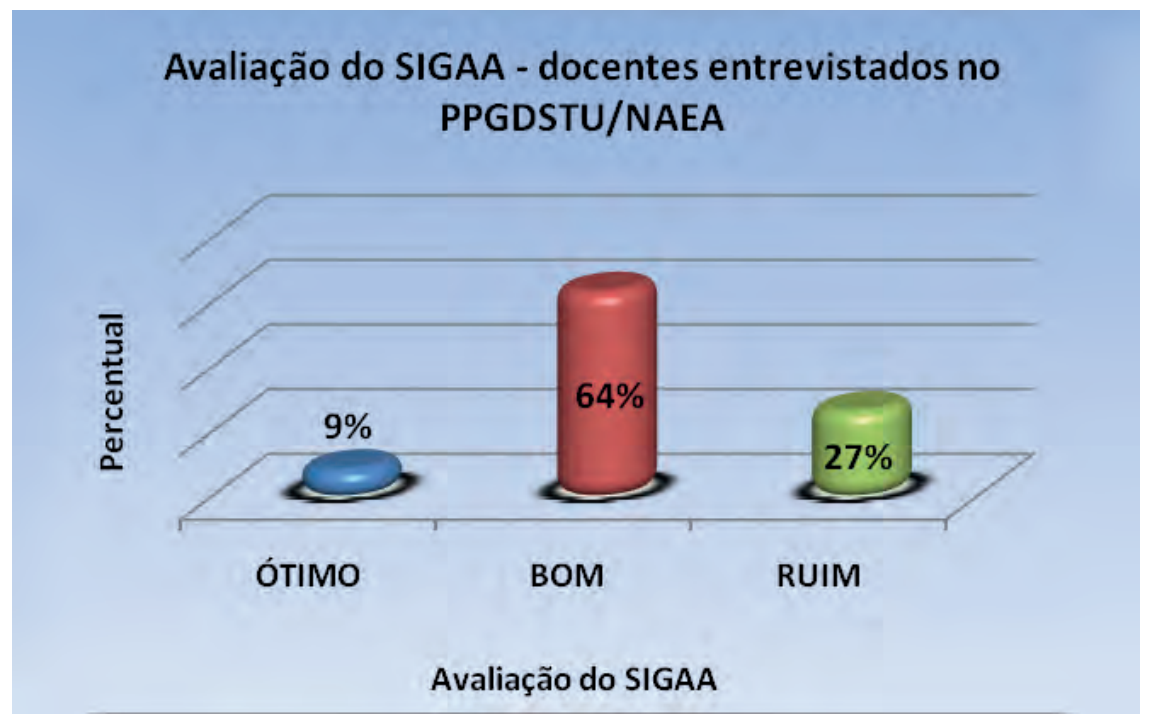

Fonte: Pesquisa de campo (2017).

Chama a atenção no Gráfico 1 o baixo percentual de docentes que consideram o SIGAA ótimo, embora a maioria o considere um bom sistema. Entretanto, quanto aos motivos alegados pelos docentes que consideram o SIGAA um bom sistema, há opiniões contraditórias, dentre as quais, cita-se a dificuldade em acessar determinadas áreas do Sistema, devendo haver alguns ajustes para o seu aprimoramento, ao mesmo tempo em que se alega a necessidade de treinamento para a melhor operacionalização da ferramenta tecnológica.

4 O código-fonte são as linhas de programação que formam um software em sua forma original e podem ser lidos por humanos (TIBONI, 2014). 
Por outro lado, um dos docentes entrevistados do PPGDSTU relatou que está satisfeito com o SIGAA, pois há "menos burocracia”, mencionando, entretanto, que "às vezes é difícil acessar". Observa-se uma relação um tanto contraditória com a ferramenta tecnológica, pois de um lado ela representa um instrumento útil ao trabalho docente, mas de outro pode se tornar quase um obstáculo a este mesmo trabalho, sendo este um dos desafios impostos pela tecnologia, especificamente ao o SIGAA.

Outra opinião de um docente que considera o SIGAA bom e que demonstra os desafios com o Sistema é a seguinte: "acho que ainda não é muito amigável e, às vezes, difícil de entender alguns caminhos para chegar no objetivo do acesso" (informação verbal) ${ }^{5}$.

Sabe-se que toda ferramenta tecnológica pode apresentar algum grau de complexidade e até de problemas quanto ao seu manuseio, ou até mesmo falhas técnicas, e parece que o SIGAA igualmente não está isento de problemas, sendo que algumas dificuldades decorrentes de sua utilização constituem alguns desafios a serem superados cotidianamente, podendo trazer impactos negativos aos usuários (VIEIRA, 2018).

Há também docentes que se consideram satisfeitos com o SIGAA, mas não o utilizam com tanta frequência, justamente quando essa ferramenta poderia ser mais explorada por eles, a fim de torná-la um canal de comunicação mais efetivo com os discentes.

Apesar de o SIGAA permitir certas facilidades e vantagens para docentes, discentes e servidores técnico-administrativos no âmbito dos PPG, a pesquisa realizada no PPGDSTU/NAEA revelou que nem todos os docentes utilizam a ferramenta tecnológica de forma adequada e a contento, visando à inserção de informações em tempo hábil para os discentes e até mesmo para o melhor fluxo administrativo do trabalho no PPG citado.

Neste sentido, o SIGAA pode se inserir na modernização referida por Silveira (2002) e Barbosa (2010), desde que todos os atores envolvidos em sua utilização colaborem para a devida inserção de informações pertinentes e necessárias às demandas de cada usuário. Deve-se ressaltar, contudo, que os portais dos programas de pós-graduação são gerados a partir das informações inseridas no sistema, o que requer que estas sejam atualizadas constantemente, inclusive pelos docentes.

Quanto aos docentes que consideram o SIGAA ruim, as opiniões são diversas, dentre as quais citam-se:

Informação concedida pelo docente entrevistado 4, em 22 de maio de 2017. 
1) pouca adequação às necessidades e demandas docentes (informação verbal) ${ }^{6}$;

2) muitas informações desnecessárias (informação verbal) ${ }^{7}$;

3) os controles não significam eficiência (informação verbal) ${ }^{8}$.

Um dos docentes do PPGDSTU opinou que “o SIGAA veio atrasar o trabalho dos pesquisadores e professores" (informação verbal) ${ }^{9}$, pois o sistema possui uma lógica muito complexa. Para este docente, os servidores técnicoadministrativos é que deveriam operar o sistema e não os docentes. No entanto, considerando que há apenas um ou dois servidores técnico-administrativos em cada PPG, seria necessário contratar mais servidores para fazer a parte que cabe ao docente no que se refere ao preenchimento do Sistema, o que poderia acarretar também mais custos com pessoal.

Especificamente no tocante aos serviços realizados por meio de sistemas informatizados como o SIGAA, todos os responsáveis pela alimentação de informações (docentes, inclusive) contribuem, no caso específico dos PPG, para o benefício dos discentes inseridos nos cursos de pós-graduação stricto sensu. Esses podem ter suas demandas resolvidas, haja vista que a interação dos discentes com os docentes e servidores técnico-administrativos, por meio do SIGAA, pode facilitar os processos de trabalho e, em última análise, pode contribuir para aumentar a eficiência da gestão pública no âmbito acadêmico e organizacional da UFPA.

Ademais, todos os trabalhadores têm seu valor e as atividades realizadas nos PPG devem cooperar sempre para o benefício de toda a sociedade, o que é corroborado por Balbe (2010), quando afirma que as TIC podem mudar a forma como a administração pública se apresenta ao cidadão contemporâneo.

No Gráfico 2, é apresentado o nível de satisfação dos docentes do PPGDSTU/NAEA com o SIGAA.

Informação concedida pelo docente entrevistado 1, em 23 de maio de 2017. Informação concedida pelo docente entrevistado 2, em 23 de maio de 2017. Informação concedida pelo docente entrevistado 3, em 25 de maio de 2017. Informação concedida pelo docente entrevistado 11, em 25 de maio de 2017. 
Gráfico 2 - Satisfação com o SIGAA - docentes entrevistados no PPGDSTU/ NAEA

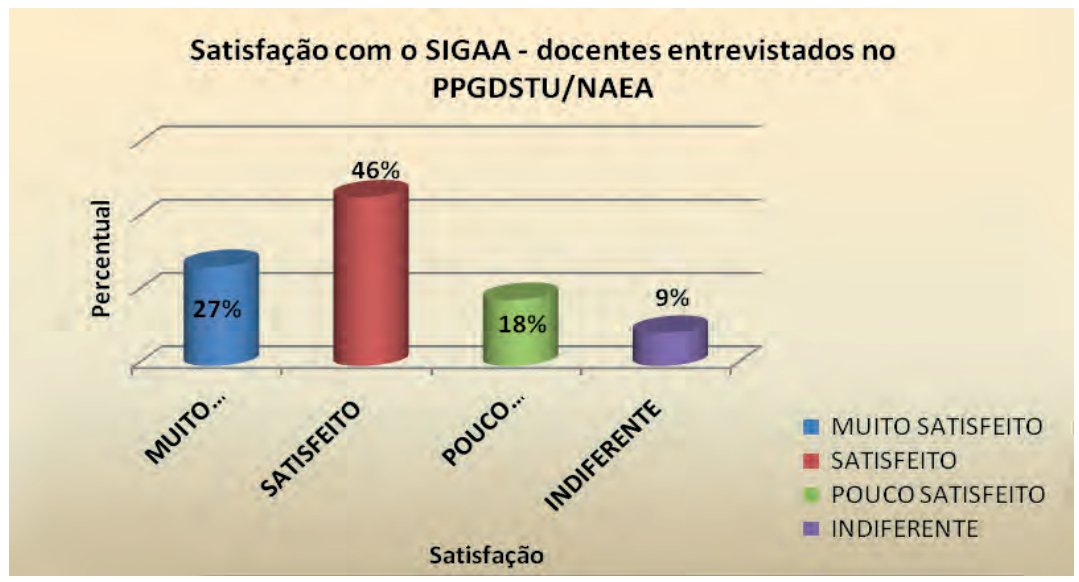

Fonte: Pesquisa de campo (2017).

Conforme se observa no Gráfico 2, o percentual de docentes do PPGDSTU/NAEA que está satisfeito com o SIGAA é de 46\%, enquanto 18\% estão pouco satisfeitos e $27 \%$ se mostraram muito satisfeitos com o sistema. Para sintetizar a opinião dos docentes, quanto às opiniões em relação à satisfação e pouca satisfação com o SIGAA, elaborou-se o Quadro 2.

Quadro 2 - Opiniões dos docentes do PPGDSTU/NAEA quanto à satisfação com o SIGAA

\begin{tabular}{|c|c|l|}
\hline Grau de satisfação & $\mathbf{\%}$ & \multicolumn{1}{c|}{ Motivos principais } \\
\hline Muito satisfeito & 30,0 & $\begin{array}{l}\text { O sistema é eficiente. } \\
\text { Atende plenamente todas as solicitações. } \\
\text { É possível resolver todas as coisas em um único sistema. }\end{array}$ \\
\hline Satisfeito & 50,0 & $\begin{array}{l}\text { Algumas áreas do SIGAA são um pouco difíceis de } \\
\text { acessar. } \\
\text { Tem atendido as demandas de docentes. } \\
\text { Está atendendo as necessidades. } \\
\text { Às vezes é difícil acessar. } \\
\text { O sistema poderia ser mais amigável. }\end{array}$ \\
\hline Pouco satisfeito & 20,0 & $\begin{array}{l}\text { Utilizado para o que os alunos necessitam (matrícula } \\
\text { em orientação e emissão de conceitos). } \\
\text { Não é instrumento necessário para o docente. } \\
\text { O sistema aumenta o trabalho docente. }\end{array}$ \\
\hline Total & $\mathbf{1 0 0 , 0}$ & - \\
\hline
\end{tabular}

Fonte: Pesquisa de campo (2017). 
Quanto ao domínio de informática dos docentes que responderam a pesquisa, a maioria possui um domínio de bom (55\%) a excelente $(9 \%)$, sendo que apenas 36\% possuem um domínio regular, o que talvez possa contribuir para a dificuldade em utilizar o SIGAA, evidenciada nas falas desses docentes conforme o Quadro 2. Neste caso, um treinamento poderia suprir essa carência, pois mesmo somando-se aqueles docentes que têm um domínio bom de informática, o percentual dos que informaram que têm dificuldade em utilizar o SIGAA foi de 55\%, contra 45\% dos que não referem dificuldade em utilizá-lo.

A totalidade dos docentes informou que não recebeu treinamento para a operacionalização do SIGAA, o que reforça mais uma vez a necessidade de treinamento constante, conforme evidenciado por alguns deles, bem como explica, em parte, a rejeição ao Sistema por um percentual de docentes entrevistados.

A respeito da frequência de utilização do SIGAA, o Gráfico 3 apresenta os resultados obtidos na pesquisa.

Gráfico 3 - Utilização do SIGAA - docentes entrevistados no PPGDSTU/NAEA

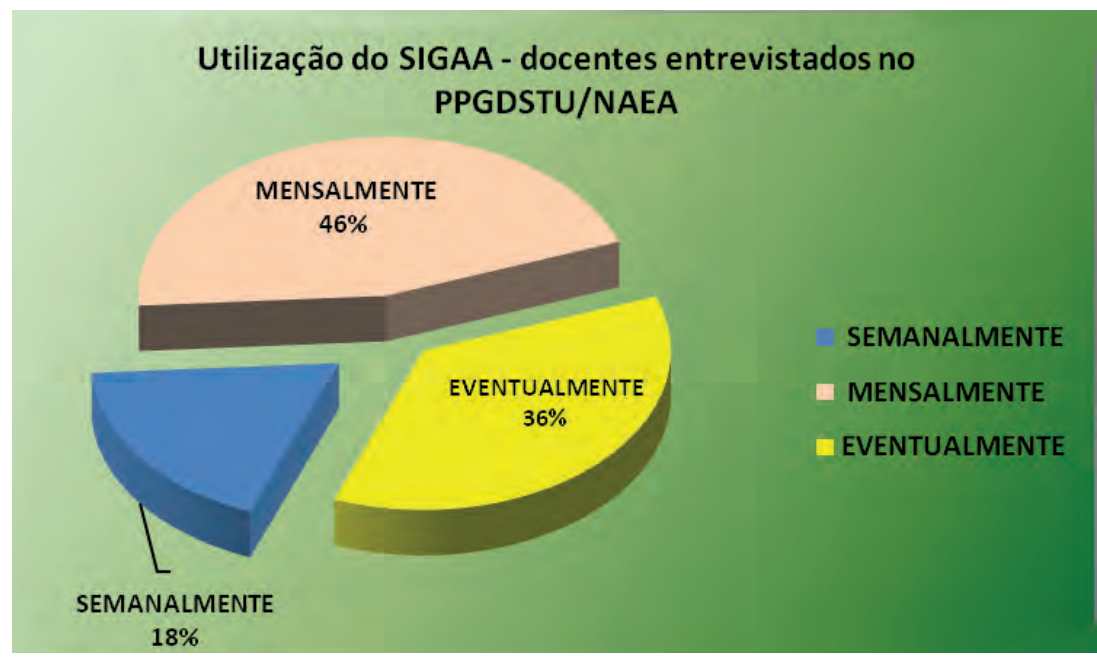

Fonte: Pesquisa de campo (2017).

Como se percebe no que se refere à frequência de utilização do SIGAA por parte dos docentes do PPGDSTU/NAEA, a maioria (46\%) o utiliza mensalmente, enquanto $36 \%$ responderam que o utilizam apenas eventualmente e somente $18 \%$ informaram que o acessam semanalmente. A razão para a baixa utilização (36\% eventualmente) se deve, segundo alguns docentes, porque o acesso ocorre de acordo com a necessidade. Outros informaram que acessam apenas quando são obrigados pelo sistema, e ainda houve menção à falta de tempo para inserção de informações no SIGAA. 
Entretanto, também entre os docentes que utilizam o sistema semanalmente e/ou mensalmente, foram relatados motivos como obrigatoriedade de utilização apenas quando impelidos pelo sistema, bem como conforme a necessidade da demanda de serviços que requerem o acesso ao SIGAA, como lançamento de conceitos, dentre outras atividades.

Apesar das críticas ao sistema e os motivos alegados para as falhas encontradas nele, $70 \%$ dos docentes do PPGDSTU/NAEA informaram que o SIGAA facilita sua vida e/ou trabalho, frente aos 30\% que disseram que o sistema não contribui para esse objetivo. Isto é evidenciado também quando a pesquisa demonstra os motivos apontados pelos docentes para que o SIGAA facilite sua vida e/ ou trabalho, conforme expresso na Tabela 1.

Tabela 1 - Sentido em que o SIGAA facilita sua vida e/ou trabalho - docentes PPGDSTU/NAEA

\begin{tabular}{|c|c|}
\hline \multicolumn{2}{|c|}{$\begin{array}{l}\text { Sentido em que o SIGAA facilita sua vida e/ou trabalho - docentes } \\
\text { PPGDSTU/NAEA }\end{array}$} \\
\hline Motivo & Percentual (\%) \\
\hline $\begin{array}{l}1 \text { - Eliminar o tempo de espera que era necessário anteriormente } \\
\text { para pedir e receber documentos no NAEA. }\end{array}$ & $29,0 \%$ \\
\hline $\begin{array}{l}2 \text { - Eliminar o custo de transporte que era necessário para se } \\
\text { deslocar para o NAEA. }\end{array}$ & $24,0 \%$ \\
\hline 3 - Aumentar a eficiência e eficácia do meu trabalho no NAEA. & $29,0 \%$ \\
\hline $\begin{array}{l}4 \text { - Reduzir erros humanos na elaboração de documentos, } \\
\text { inclusive declarações. }\end{array}$ & $18,0 \%$ \\
\hline Total & $100,0 \%$ \\
\hline
\end{tabular}

Fonte: Pesquisa de campo (2017).

Fica claro, contudo, que o SIGAA, enquanto ferramenta tecnológica para modernização das atividades em âmbito acadêmico e administrativo na UFPA no contexto dos PPG, é um sistema que, mesmo tendo determinados problemas, limitações e desafios a serem superados, é avaliado positivamente pela maioria dos docentes do PPGDSTU/NAEA.

No que tange às recomendações dos docentes do PPGDSTU/NAEA para a melhoria da utilização do SIGAA, as seguintes contribuições foram citadas:

1) Treinamento constante de todas as categorias para melhor inserção de informações e diminuição de erros;

2) Adequá-lo às necessidades dos docentes;

3) Adaptá-lo com informações básicas;

4) Pensá-lo a partir da realidade da UFPA;

5) Ajustá-lo a outros cadastros, como CNPq, CAPES, Lattes etc.; 
6) Melhorar os controles de acesso;

7) Importação para o SIGAA dos dados da Plataforma Sucupira em forma textual, permitindo a utilização em outras atividades relativas à docência e pesquisa;

8) Aprimoramento do SIGAA para melhoria de sua operacionalização;

9) Deixar de ser operado pelo docente e delegar essa atividade ao servidor técnico-administrativo.

As recomendações propostas refletem a opinião dos docentes entrevistados, tanto dos que são favoráveis ao SIGAA, quanto daqueles que não gostam de lidar com essa ferramenta tecnológica. Todas as contribuições são válidas, entretanto, verifica-se que a proposta do SIGAA é ser uma ferramenta auxiliar ao trabalho de cada categoria (docentes, discentes e servidores técnico-administrativos), cada um com seu perfil de trabalho e utilização.

No entanto, uma vez que a senha para acesso ao sistema é pessoal e intransferível, cabe ao docente, por exemplo, utilizando seu perfil de acesso, inserir as informações em tempo hábil para que o discente possa encontrar essa informação quando dela necessitar. Assim, cada um fazendo sua parte contribui para a melhoria dos processos de trabalho no âmbito de cada PPG. Por outro lado, mais uma vez se reforça a necessidade de treinamento para melhor utilização do SIGAA e para a diminuição de equívocos de modo geral, conforme proposto por parte dos docentes entrevistados no PPGDSTU.

\subsection{DO CORPO DISCENTE}

$\mathrm{Na}$ pesquisa, os discentes do PPGDSTU/NAEA, em sua maioria, se mostraram satisfeitos com o SIGAA; 82\% avaliaram o sistema como bom e 18\% como ótimo. Nenhum discente referiu que o SIGAA é ruim, o que demonstra uma ampla aceitação ao sistema.

Entretanto, mesmo avaliando positivamente o sistema, quando indagados o quanto estavam satisfeitos com o SIGAA, 9,1\% dos discentes do PPGDSTU/ NAEA referiram que não estão satisfeitos, ante $90,9 \%$ que referiram que estão satisfeitos, considerando os dados agregados (Quadro 3).

O Gráfico 4 mostra os resultados obtidos em relação ao nível de satisfação com o SIGAA por parte dos discentes entrevistados no PPGDSTU. 
Gráfico 4 - Satisfação com o SIGAA - discentes entrevistados no PPGDSTU/ NAEA

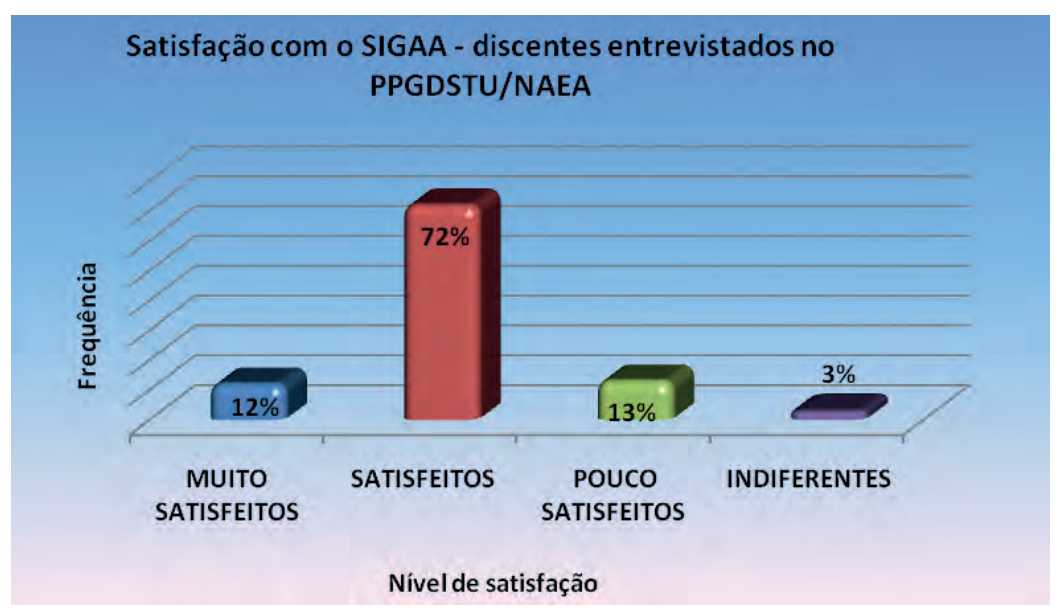

Fonte: Pesquisa de campo (2017).

Conforme se verifica nos números expressos no Gráfico 4, apenas 13\% dos discentes se mostraram pouco satisfeitos, mas a ampla maioria dos alunos (84\%) se mostraram, respectivamente, muito satisfeitos e satisfeitos com o SIGAA, sendo o percentual de indiferentes de apenas 3\%. Os discentes que se mostraram satisfeitos com o sistema apresentaram razões variadas para isso, citando desde a facilidade em utilizá-lo, não tendo problemas quanto ao acesso, bem como pelo fato de o SIGAA ser um sistema de fácil aprendizado em que o discente pode aprender suas funcionalidades intuitivamente, ao mesmo tempo em que sua característica on-line permite a economia de recursos e evita o deslocamento para a resolução de determinadas questões que o sistema permite.

Entretanto, apesar de estarem satisfeitos com o SIGAA e terem respondido que o sistema é bom ou ótimo, alguns discentes referiram determinados problemas com o sistema, dentre os quais se destaca: “[...] precisa melhorar em alguns aspectos. A plataforma é um pouco complicada de entender como funciona, tinha que ser mais direto e claro os campos de informações" (informação verbal) $)^{10}$.

Por este comentário, percebe-se que se houvesse treinamento para que os discentes entendessem melhor as funcionalidades do SIGAA, o sistema poderia ser mais bem explorado por eles. A mesma opinião foi emitida por um docente do PPGDSTU, ao afirmar que deveria ser mais fácil a navegação no SIGAA. Também é natural se esperar que um sistema de informações seja mais eficaz

10 Informação concedida pelo discente entrevistado 4, em 29 de maio de 2017. 
ao simplificar as informações geradas ao usuário, pois isso demandará menos trabalho e conferirá mais agilidade em sua operação.

Mencionou-se de forma recorrente também o seguinte: "as informações disponíveis nem sempre estão atualizadas" (informação verbal) ${ }^{11}$ e, recorrentemente, que "o sistema deve permitir maior interação com outros usuários" (informação verbal) ${ }^{12}$. Com efeito, os sistemas de informação precisam ser constantemente atualizados para cumprir o seu papel enquanto ferramenta auxiliar ao trabalho humano. Neste sentido, no que tange especificamente aos discentes, a atualização de informações por parte dos docentes é um ponto fundamental e necessário para que os discentes tenham as informações demandadas em tempo hábil.

Foi mencionado por outros discentes, de forma recorrente, que o sistema "é subutilizado", destacando-se o aspecto da ausência de interação virtual, fato que "causa uma dificuldade de colaboração acadêmica" (informação verbal) ${ }^{13}$. Ressalta-se o aspecto da interação entre docentes e discentes por meio do SIGAA, devendo haver, por parte dos docentes, a devida inserção de informações pertinentes à vida acadêmica dos discentes, bem como uma maior e mais frequente utilização das ferramentas disponíveis no Sistema para tal finalidade.

Outro discente destacou a necessidade de que os documentos emitidos pelo SIGAA venham com uma assinatura eletrônica da Coordenação do PPG, a fim de evitar que os alunos tenham que se deslocar até a Secretaria do PPG, uma vez que muitas instituições exigem a assinatura e carimbo do coordenador ou coordenadora do programa de pós-graduação ao qual o discente é vinculado.

Isto vai de encontro ao objetivo do SIGAA, considerando que é uma ferramenta tecnológica pensada para propiciar praticidade aos usuários, conforme corroborado por autores como Barbosa (2010) e Silveira (2002). Neste caso, as empresas e outros órgãos públicos parecem desconhecer o SIGAA, os tipos de documentos que o sistema fornece e a autenticidade destes, bem como o fato de que há um número de autenticidade presente nos documentos emitidos pelo SIGAA.

A frequência de utilização do SIGAA por parte dos discentes do PPGDSTU/NAEA é expressa no Gráfico 5.

\footnotetext{
Informação concedida pelo discente entrevistado 12, em 29 de maio de 2017. Informação concedida pelo discente entrevistado 11, em 30 de maio de 2017.

13 Informação concedida pelo discente entrevistado 33, em 31 de maio de 2017.
} 


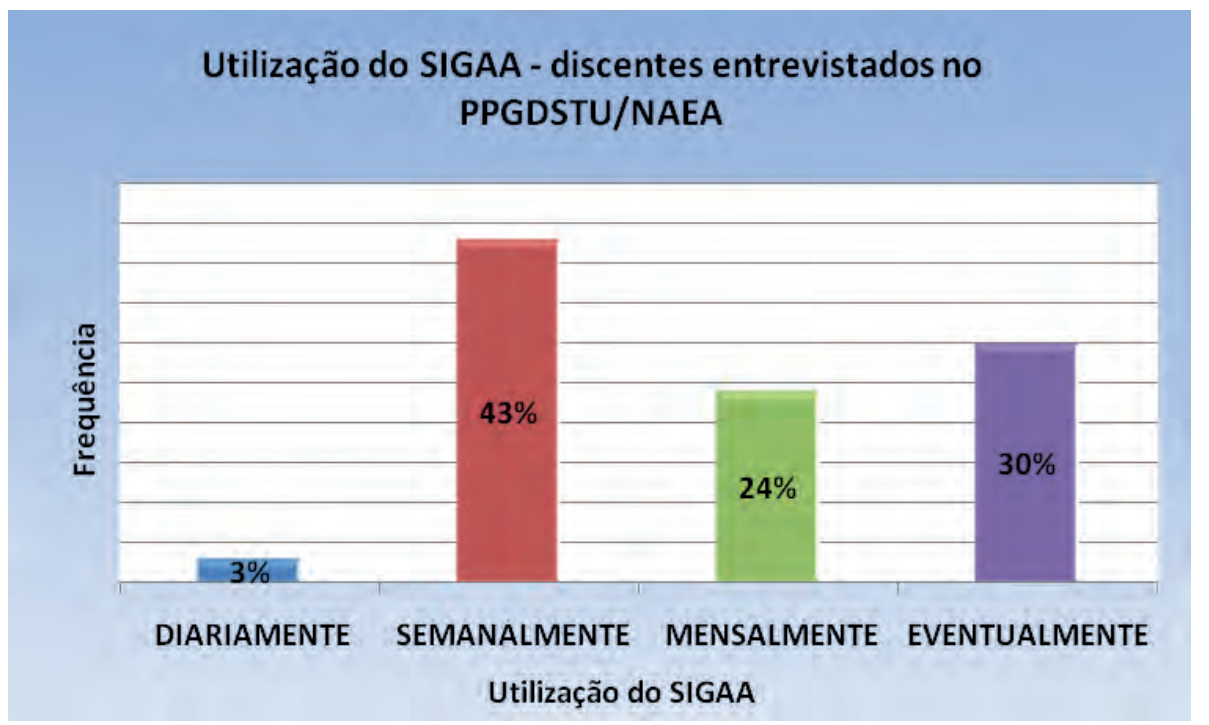

Fonte: Pesquisa de campo (2017).

Percebe-se que os discentes, em sua maioria (43\%), utilizam o SIGAA semanalmente para visualizar conceitos, emitir histórico e declarações. Contudo, o sistema poderia ser mais utilizado por um número maior de discentes, caso fossem inseridas mais informações pertinentes ao curso que realizam. Essa falta de informações atualizadas pode contribuir, em parte, para que uma parcela significativa de discentes (30\%) utilizem o SIGAA apenas eventualmente.

Além disso, o SIGAA pode, por exemplo, contribuir para a preservação do meio ambiente, uma vez que o discente pode simplesmente visualizar seu histórico ou outra informação sem a necessidade de imprimir o documento, reduzindo a quantidade de papel, contribuindo, assim, para uma sociedade sustentável. Ao mesmo tempo, a administração pública e especificamente os PPG dos quais os discentes fazem parte também se beneficiam, pois diminuem ou, em alguns casos, eliminam a utilização de papel, contribuindo para a mitigação da degradação do meio ambiente e para a existência de processos de trabalho mais sustentáveis.

Quanto à questão se o SIGAA facilita a vida e/ou trabalho dos discentes, 94\% dos entrevistados afirmaram que "sim", sendo que apenas $6 \%$ os discentes informaram que o SIGAA "não" contribui para tal finalidade.

Indagados sobre os motivos pelos quais o SIGAA facilita a vida e/ou trabalho dos discentes do PPGDSTU/NAEA, os mais citados na entrevista foram que o sistema, por ser on-line, permite que muitas demandas sejam realizadas 
a distância, sem necessidade de comparecimento presencial à Secretaria do PPG e ter que esperar algum tempo para a resolução da demanda, ensejando ainda a economia de recursos financeiros.

Ao mesmo tempo, com o SIGAA é possível se evitar o dispêndio de dinheiro com transporte e ainda de tempo, pois a característica fundamental de um sistema de informação que funciona via internet é a de prover acesso imediato às informações, conferindo praticidade ao dia a dia do cidadão.

Houve menção também ao fato de que com o SIGAA alguns processos se tornaram menos burocráticos, conferindo mais autonomia aos discentes em seu dia a dia, posto que eles podem resolver suas questões apenas com o auxílio do Sistema, pelo fato de que a informação se torna mais ágil, precisa e transparente.

No PPGDSTU/NAEA, a maioria dos discentes apontaram que o SIGAA facilita sua vida e/ou trabalho. No entanto, apesar de haver críticas quanto à função e à importância do Sistema, houve discente do PPGDSTU que referiu que ele facilita a vida, pois "o SIGAA se apresenta enquanto ferramenta importante ao diálogo entre professor e alunos" (informação verbal) ${ }^{14}$.

Por outro lado, os discentes do PPGDSTU que se manifestaram contrariamente alegaram que o SIGAA, para facilitar, de fato, a vida dos discentes, "precisa conter informações atualizadas e estar conectado às demandas enviadas por e-mail' (informação verbal) ${ }^{15}$, considerando que muitas informações são enviadas diretamente por e-mail aos discentes e não são inseridas no Sistema.

A Tabela 2 sintetiza, segundo a pesquisa, em que sentido os discentes têm sua vida facilitada pelo SIGAA.

Tabela 2 - Sentido em que o SIGAA facilita sua vida e/ou trabalho - discentes PPGDSTU/NAEA

\begin{tabular}{|c|c|}
\hline Motivo & $(\%)$ \\
\hline $\begin{array}{l}\text { 1) Eliminar o tempo de espera que era necessário anteriormente para pedir e } \\
\text { receber documentos no NAEA. }\end{array}$ & $43,0 \%$ \\
\hline $\begin{array}{l}\text { 2) Eliminar o custo de transporte que era necessário para se deslocar para o } \\
\text { NAEA. }\end{array}$ & $26,0 \%$ \\
\hline 3) Aumentar a eficiência e eficácia do meu trabalho no NAEA. & $15,0 \%$ \\
\hline 4) Reduzir erros humanos na elaboração de documentos, inclusive declarações. & $16,0 \%$ \\
\hline Total & $100,0 \%$ \\
\hline
\end{tabular}

Fonte: Pesquisa de campo (2017).

14 Informação concedida pelo discente entrevistado 28, em 31 de maio de 2017

15 Informação concedida pelo discente entrevistado 17, em 31 de maio de 2017. 
Acerca das facilidades proporcionadas pelo SIGAA, a maioria dos discentes do PPGDSTU/NAEA (43\%) se referiram ao item eliminar o tempo de espera que era necessário anteriormente para pedir e receber documentos no NAEA.

Tais opiniões corroboram o caráter de agilidade que os sistemas de informação conferem à vida dos indivíduos, tal como o SIGAA, que, por ser on-line os usuários (discentes, docentes e técnico-administrativos) resolvem as questões com agilidade, desde que esteja funcionando normalmente, considerando que a função principal de toda e qualquer tecnologia é aperfeiçoar as condições de vida ou de trabalho de um ou de vários indivíduos, conforme evidenciado por Pinto et al. (2019).

Sobre as recomendações para melhoria da utilização do SIGAA, os discentes do PPGDSTU/NAEA listaram as seguintes, expostas no Quadro 3.

Quadro 3 - Recomendações para melhoria da utilização do SIGAA, segundo os discentes do PPGDSTU/NAEA

1) Criação de um espaço virtual de entrega de trabalhos acadêmicos, com a geração de QR Code ou de outros mecanismos de segurança e confiabilidade, para tornar mais prática a relação docente/discente.

2) Criação de um espaço que aglutine e ofereça tempestivamente aos alunos indicações de editais nacionais e internacionais abertos para a publicação de trabalhos científicos.

3) Resolver os problemas de lentidão e não atualização das informações dos discentes que ocorrem com frequência, bem como solucionar erros referentes a situações dos discentes.

4) Divulgação dos recursos ofertados pelo sistema, inclusive com treinamento para os discentes no início dos cursos, para aprender sobre o que é e como funciona o SIGAA e todas as suas ferramentas, bem como treinamento para os docentes, para que se influencie na cultura de utilizar as ferramentas oferecidas pelo sistema.

5) Maior interação por parte dos docentes e lançamentos de materiais e informações no SIGAA sob sua responsabilidade.

6) Ter mais opções para solicitação de documentos, aumentando a transparência das atividades desenvolvidas na UFPA.

7) Emissão dos documentos com uma assinatura eletrônica da coordenadora ou coordenador de Programa e que permitam que haja um espaço de orientação entre orientando e orientador, para que alguns trabalhos possam ficar salvos, recomendações de leituras, um local com calendário para reuniões entre ambos e utilização do fórum de debates.

8) O acesso ao que se quer poderia ser mais facilitado, com os caminhos bem delineados e explícitos. Ser totalmente livre e que todos possam ter acesso, aplicando o princípio da transparência em todos os sentidos.

9) Adicionar módulos que permitam maior integração com outras plataformas ou aplicativos (nuvem, e-mail, aplicativos como Whats App, SMS).

10 ( Simplificação do SIGAA, evitando duplicidade de informações.

11) Inserção de informações a respeito de submissão e controle de projetos e bolsas de pesquisa, submissão e controle de ações de extensão, submissão e controle dos projetos de ensino (monitoria e inovações), atividades de ensino a distância no ambiente virtual de aprendizagem e atualização das informações referentes a bolsas de pesquisa.

Fonte: Pesquisa de campo (2017). 
As recomendações constantes no quadro acima visam melhorar a interação entre docentes e discentes e, em última análise, aprimorar a gestão pública e o gerenciamento acadêmico, bem como facilitar a vida dos usuários, levando também a uma melhor utilização do SIGAA por parte de todos os que estão inseridos no dia a dia dos programas de pós-graduação stricto sensu.

\section{CONSIDERAÇÕES E RECOMENDAÇÕES}

No que concerne à contribuição do SIGAA para o gerenciamento do programa de pós-graduação stricto sensu analisado neste trabalho, aliado aos achados na literatura, a pesquisa aponta que ele é um sistema que tem se mostrado útil para esse gerenciamento, tanto em âmbito acadêmico quanto administrativo.

Nesta perspectiva, a pesquisa realizada com docentes, discentes e servidores técnico-administrativos neste estudo permitiu concluir como pontos convergentes que as três categorias analisadas entendem que o SIGAA pode contribuir para a melhoria dos processos de trabalho no PPGDSTU/ NAEA. Deve haver, entretanto, conforme ficou evidenciado, maior incentivo institucional ao uso do sistema e maior adesão por parte de alguns docentes quanto à inserção de informações atualizadas para os discentes. Isso pode indicar que os PPG e a UFPA, de modo geral, precisam aprimorar as estratégias para divulgação e maior aceitação do SIGAA entre todas as categorias analisadas neste estudo. Por outro lado, a pesquisa revelou que a maioria dos usuários entrevistados percebem o SIGAA como um sistema útil para o gerenciamento acadêmico e administrativo dos PPG.

Recomenda-se, assim, que haja treinamento permanente para docentes e discentes, a fim de que o SIGAA seja mais utilizado no cotidiano dos usuários inseridos no PPGDSTU, para se tirar maior benefício desse sistema, bem como para o alcance dos objetivos estratégicos e de modernização da gestão que a UFPA persegue. Defende-se também, por parte dos docentes, a constante atualização de informações, a fim de que os discentes e o público externo ao PPG possam se informar das atividades docentes.

Como recomendações, foram reveladas questões como a necessidade de treinamento, principalmente para docentes e discentes, objetivando aumentar a adesão e o uso do SIGAA, e o maior engajamento entre estes, para se tirar maior benefício desse sistema. Neste contexto, defende-se a necessidade de que o PPG analisado neste trabalho implemente como política institucional interna ações voltadas para a conscientização de docentes quanto à utilização do SIGAA de maneira mais efetiva, objetivando a interação com os discentes por meio da 
Turma Virtual, recurso presente no sistema, mas pouco utilizado, conforme ficou evidenciado nesta pesquisa. Essa foi uma das constatações relatadas inclusive por parte de alguns discentes, ao apontarem que o SIGAA é subutilizado devido justamente não ser adequadamente usado por alguns docentes.

Evidencia-se, portanto, como sugestão aos PPG e à própria UFPA, a oferta de treinamento permanente a docentes e discentes, aliado a um plano institucional de comunicação acerca da necessidade de utilização do SIGAA por parte de todos os docentes, o que pode contribuir para uma mudança cultural e comportamental no que concerne à aceitação do SIGAA para melhorar o gerenciamento do curso de pós-graduação stricto sensu analisado nesta pesquisa e, de modo mais amplo, para a Universidade Federal do Pará.

Como recomendação ao CTIC/UFPA, tem-se o aprimoramento do SIGAA com vistas à integração com Currículo Lattes, Plataforma Sucupira, dentre outras plataformas, bem como trabalhar na maior simplificação do sistema e atualização constante dos manuais do SIGAA, tendo em vista que alguns usuários podem não ter tanta facilidade em manusear determinadas ferramentas tecnológicas. Isso, talvez, poderia contribuir para uma utilização e interação mais ampla dos usuários do sistema. As contribuições desta pesquisa podem ser ampliadas e exploradas em trabalhos futuros, tendo-se como temas as TIC, a gestão pública e os aspectos organizacionais das universidades públicas no Brasil.

\section{REFERÊNCIAS}

BALBE, R. S. Uso de tecnologias de informação e comunicação na gestão pública: exemplos no governo federal. Revista do Serviço Público, Brasília, v. 61, n. 2, p. 189-209, abr./jun. 2010.

BARBOSA, A. F. Pesquisa sobre o uso das tecnologias da informação e da comunicação no Brasil: TIC governo eletrônico - 2010. São Paulo: Comitê Gestor da Internet no Brasil, 2010.

BURIGO, C. C. D. et al. Mestrado Profissional em Administração Universitária: desafios e perspectivas no processo da gestão universitária. Revista de Gestão e Tecnologia Navus, Florianópolis, v. 6, n. 2, p. 68-78, abr./jun. 2016. Disponível em: http://navus.sc.senac.br/index.php/navus/article/view/323. Acesso em: 29 out. 2020.

CASTELLS, M. A sociedade em rede. São Paulo: Paz e Terra, 1999.

CRESWELL, J. W. Projeto de pesquisa: métodos qualitativo, quantitativo e misto. 2. ed. Porto Alegre: Artmed, 2007. 
DIAS, I. M. A relação entre reforma da administração pública e tecnologias de informação no governo do estado de São Paulo. 2008. 170 f. Tese (Doutorado em Administração) - Programa de Pós-Graduação em Administração, Faculdade de Economia, Administração, Contabilidade e Atuária, Universidade de São Paulo, São Paulo, 2008.

DÍAZ, D. C. et al. Sistemas integrados em uma universidade brasileira: informação cooperada como recurso estratégico. Universidad de Málaga, 03. jul. 2014. Disponível em: http://hdl.handle.net/10630/7762. Acesso em: 23 ago. 2016.

DINTOE, S. S. Information and communication technology use in higher education: Perspectives from faculty. International Journal of Education and Development using Information and Communication Technology (IJEDICT), [S. l.], v. 14, n. 2, p. 121-166, 2018.

FARIAS FILHO, M. C.; VILHENA, M. G.; NASCIMENTO, D. M. Gestão de processo na implantação de um sistema de informação acadêmica: a experiência da UFPA. Revista GUAL, Florianópolis, v. 7, n. 2, p. 69-85, maio 2014.

NÚCLEO DE ALTOS ESTUDOS AMAZÔNICOS. Histórico da entidade. Núcleo de Altos Estudos Amazônicos, Belém, 2017. Disponível em: http:// www.naea.ufpa.br/index.php/institucional-apresentacao. Acesso em: 02 jan. 2017. PINTO, A. L. S. et al. Avaliação da aceitação das ferramentas tecnológicas no ambiente do trabalho docente. Revista GUAL, Florianópolis, v. 12, n. 2, p. 118 138, maio/ago. 2019.

PORCIUNCULA, C. S. A modernização tecnológica no setor público: a experiência de cooperação. Informática Pública, Porto Alegre, ano 2, n. 1, p. 31-35, 2001. Disponível em: www.ip.pbh.gov.br/ANO2_N1_PDF/ ip0201 porciuncula.pdf. Acesso em: 19 maio 2015.

PORTO, R. M. A. B; MAGALHÃES, A. M. Gestão da informação como suporte à redução de incerteza no processo decisório: estudo de caso do Instituto Federal de Minas Gerais - IFMG. Informação \& Tecnologia (ITEC), Marília/João Pessoa, v. 1, n. 1, p. 81-95, jan./jun. 2014.

ROCHA NETO, A. F.; LIMA, G. A. F. Turma virtual do SIGAA como ferramenta de apoio ao ensino. Natal: UFRN, 2009.

SILVEIRA, S. A. Governo eletrônico e inclusão digital. In: HERMANNS, K. (org.). Governo eletrônico: os desafios da participação cidadã. Fortaleza: Fundação Konrad Adenauer, 2002. p. 69-81. Disponível em: https://bvc.cgu. gov.br/bitstream/123456789/3350/1/Arquivos_transparencia_do_estado_ capcidade.pdf. Acesso em: 25 ago. 2016. 
TIBONI, A. C. Software livre como política de governo. 2014. 67 f. Monografia (Especialização em Gestão Pública) - Escola de Administração, Universidade Federal do Rio Grande do Sul, Porto Alegre, 2014.

UNIVERSIDADE FEDERAL DO PARÁ. UFPA em números. Universidade Federal do Pará, Belém, 2016. Disponível em: http://www.ufpanumeros.ufpa. br/. Acesso em: fev. 2018.

UNIVERSIDADE FEDERAL DO PARÁ. Manual do Usuário SIG. Belém: CTIC/UFPA, 2011. Disponível em: https://portal.ufpa.br/images/docs/ sigufpa/manuais/manual_sig_autocadastro.pdf. Acesso em: 22 set. 2015.

VIEIRA, J. N. Tecnologia da Informação e Comunicação (TIC) na gestão da pós-graduação interdisciplinar da UFPA: um estudo de caso sobre o Sistema Integrado de Gestão de Atividades Acadêmicas (SIGAA). 2018. 189 f. Dissertação (Mestrado em Planejamento do Desenvolvimento) - Programa de Pós-Graduação em Desenvolvimento Sustentável do Trópico Úmido, Núcleo de Altos Estudos Amazônicos, Universidade Federal do Pará, Belém, 2018.

VIEIRA, J. N.; CORBIN, H. P. As possibilidades das Tecnologias da Informação e Comunicação (TIC) como ferramentas para gestão pública da Universidade Federal do Pará. In: PEZZUTI, J.; AZEVEDO-RAMOS, C. (org.). Desafios amazônicos. 1. ed. Belém: NAEA, 2016. p. 225-260. (Série Desenvolvimento e Sustentabilidade).

VILHENA, M. G. S. Organização institucional da UFPA no contexto de reforma do Estado: uma análise dos modelos de gestão e processos na implantação do SIE (2005 a 2009). 2011. 128 f. Dissertação (Mestrado em Planejamento do Desenvolvimento) - Programa de Pós-Graduação em Desenvolvimento Sustentável do Trópico Úmido, Núcleo de Altos Estudos Amazônicos, Universidade Federal do Pará, Belém, 2011.

YOSHINO, C. K. N. Fatores críticos de sucesso como antecedentes da aceitação de um sistema de informação em uma universidade federal. 2010. 135 f. Dissertação (Mestrado em Administração) - Centro de Ciências Sociais Aplicadas, Universidade Federal do Rio Grande do Norte/Universidade Federal do Pará, Natal, 2010.

YOSHINO, C. K. N.; RAMOS, A. S. M. Fatores críticos de sucesso como antecedentes da intenção comportamental de usar sistemas ERP: um estudo empírico. Gestão e Desenvolvimento, Novo Hamburgo, v. 12, n. 1, p. 87-104, jan. 2015.

Texto submetido à Revista em 01.07.2020

Aceito para publicação em 01.12.2020 
